\title{
Subspace Clustering Algorithm Based on Multi-rule Constraint
}

\author{
Huiping Li \\ Bao Tou Medical College \\ Inner Mongolia Bao Tou 014040, China \\ lhpccc@163.com
}

\begin{abstract}
For the fact that telecom data size is extremely huge and the management is much complicated, the paper proposes subspace clustering algorithm based on mult-rule constraint, to mine business knowledge information in a more efficient and a curate manner. By relying on K-means clustering algorithm, the method improves selection and mutation operation of genetic algorithms and thus corrects inappropriate choice of K-means initial clustering centers. Meanwhile, with the use of variable weighting strategy, data classification sparseness in the clustering is overcome. A fast and useful mining method is enabled for massive data. Results show its better performance in terms of computilg efficiency, accuracy and ability.
\end{abstract}

Keywords: data mining, telecommunications drea, $k$-means, cloud computing

\section{Introduction}

Another challenge accompanied with the information society is occurrence of tremendous data every hour and moment. Those datan play a critical role in company operation and business decision. As statisties suggest the bill volume of China Mobile every single day reaches $1 \mathrm{~TB}$ for a prefecture-level city. Google needs to process worldwide webpages every day for over 20PB. Those data, if used effectively, will bring about unlimited values to enterprises. If they re neglected or of disregard, they'll become "data tombs", which would add enterprise operational costs [1-5]. There will have difficulties when enormous data are being discovered:

When data volume is considerably huge, tele-data up to hundreds of T-scale memory size is commonly seen [6-77. In this case, it will extremely hard to perform knowledge discovery through traditional data mining tools. More unexpected problems will occur if complicated application tasks and enterprise requirements are combined together. To be specific, in normal running cases, programs will abruptly terminate and resources collapse, which are both anticipated [8-9].

More sophisticated processing methods and systematic planning are required. It's necesary to consider in a global sense the integrative performance of clustering algorithms [10-11]. In the process of treatment, lots of practical issues should be settled like: if the knowledge classification is appropriate; if business knowledge is accurate; how to achieve positively the best rate of precision and acquire business knowledge satisfactory to users [1213].

To sum from the above, data size is inversely related to the performance of methods. With size growing, the comprehensive performance of methods will degrade linearly. It's required to strengthen the effectiveness of data mining techniques by plenty of rule and constraint mechanisms. Hence for the size of tele-data, it uses the global optimal features of genetic 
algorithms and precise subspace clustering characteristics of variable weighting scheme to modify traditional K-means clustering methods, for a proper data mining technique which has better overall performance [14-15].

\section{Application and Analysis of the Method}

The subspace clustering is composed of four parts. To make it clear, we'll introduce the design and implementation procedure of it by taking the case of dynamic classification of telecom product suppliers.

Retrieve historical behavior records of those providers to prepare source data of that dynamic categorization;

Date pre-treatment, carry out normal operation of data to form features for describing behavioral data and establish the matrix;

According specific knowledge discovery demand and data features, the dynamic classifying strategy is developed on the basis subspace clustering of variable eighting;

Compare dynamic and static classification results as to adjust tefecom operational strategies.

Behavior characteristic characterization: Conventional classifying methods use qualitative index and inherent attributes as major evaluation basis, without regard of behavior's internal association and interaction between telecom enterprises and suppliers. As plentiful features change along with trade volume, errors are directly caused in the discovery of final knowledge.

Data matrix: As time axis defines, tele-data can be described to behavior attribute set, i.e., basic information of articles in every singie given time range. Each to-be-discovered target can be expressed to a value set of those attributes. The whole target set can be expressed to a $\mathrm{N} x$ M matrix, where $\mathrm{N}$ is total number of targets and $\mathrm{M}$ is totality of behavior attribute. Table I lists the behavior data matrix of one supplien

Object data is divided into $C_{0}$ and $C_{1}$. The behavioral pattern of targets in both groups is exactly similar. Column rere to behavior attribute of objects; 0 and 1 respectively means the trade behavior which doesn't happentand has occurred.

\begin{tabular}{|l|l|l|l|l|l|}
\hline \multicolumn{7}{l}{ Table 1. Behavior Data Matrix } \\
\hline Attribute & & & & \\
\\
Grouping
\end{tabular}

In real situation, the matrix is of tremendous size. When it has high dimensions, data get much sparser. So for effective data mining, it's necessary to adopt subspace clustering 
analysis method based on variable weighting strategy.

Variable weighting K-means subspace clustering method can meet knowledge discovery demands by overcoming hi-dimension, huge amount and data sparseness. With modification of data variable weight, there will be importance and less importance for different objects, instead of mere deletion or conservation. Furthermore, the method retains data processing ability of traditional K-means mining, especially suitable for discovery of bulky data like telecom information. The application of data behavior matrix can describes effectively the attribute information of various telecom business types, rather fit for mining data in the telecom field.

Set Data matrix with $\mathrm{N} \times \mathrm{M}$ contains $\mathrm{M}$ variable, the $\mathrm{N}$ object is divided into $\mathrm{K}$ type, set $\Re_{i}=\left(\delta_{i, 1}, \delta_{i, 2}, \ldots, \delta_{i, m}\right)$ the weight of variable in the $i-t h$ class; set $\mathfrak{R}=\left(\Re_{1}, \Re_{2}, \ldots, \Re_{K}\right)$ the weight collection of all variables in all $\mathrm{K}$ classes.

The variable weighted subspace clustering method in the calculation process automatically adjusts the variable weight of each cluster value. In order to meet the data needs, to reduce the classification problem of sparse impact on the clustering results. Formulation is expressed as:

$$
F(W, Z, \Re)=\sum_{l=1}^{K} \sum_{j=1}^{N} \sum_{i=1}^{M} w_{l, j} \delta_{l, j}{ }^{\beta}\left[d\left(z_{T, j}, C_{i, i}\right)+\eta\right]
$$

As mentioned earlier, $\mathrm{K}$ is the number of clusters, $\left[W_{i, j}\right]$ is an integer matrix of $\mathrm{K} \times \mathrm{N}$. The variable $\mathrm{Z}$ represents a $\mathrm{K}$ cluster senter, $\mathrm{Z}$ is the $\mathrm{K} \times \mathrm{M}$ integer matrix elements. $d\left(z_{l, j}, x_{j, i}\right)$ represents object $j$ to clustercenter $l$ distange at $i-t h$ characteristic

As mentioned before, in order to overcome the data sparseness problem, once in the weight calculation. Introduced a steady 9 constant $\varepsilon$. In subspace clustering, in order to facilitate the processing of feature weights, introduces a parameter $\eta$. When $\eta$ is too large, the algorithm will $\mathrm{K}$ back to the standard K-means clustering algorithm. When $\eta$ is too small, the whole sample space will focus on the sparse higher degrees of variables, while neglecting other important variables "Therefore, selecting parameter $\eta$ take specific manner, in general, meeting the following conditions.

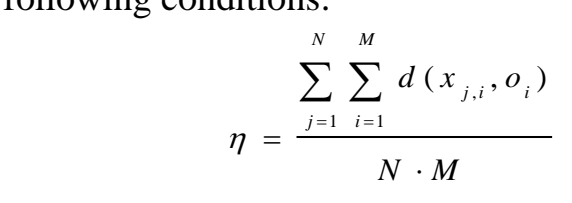

Where, $o_{i}$ represents characteristics average of the whole sample space. For the application of mass data, complex, it can be sampled to determine the parameter $\eta$ size. After discussed, for the business application of this case, it can be sampled $20 \%$ to obtain satisfactory clustering results.

It solves data processing problems between data objects and in the process of knowledge discovery, capable to analyze and treat data dynamic classification. In the context of data exchange, K-means methods have the feature of rapid treatment of abundantly huge data. Subspace clustering methods can take advantage of it to process in a more efficient way hidimension data and completely get rid of the ubiquitous knot of data sparsity. 


\section{Experiment Design and Discussion}

\subsection{Test Scenario and Data}

The test environment here is on VC.NET application to perform synchronous test. Crank call information mining was chosen as primary data for business analysis and validation. Still, comparison was made among several characteristics like algorithm computing efficiency, to determine the comprehensive performance of the subspace clustering algorithm based on multi-rule constraint. Crank call information mining is the process of all suspected strange call numbers to be accurately searched from tremendous telecom communication data, which solved in essence the actual situation that customer service staffs have to dial back to confirm nuisances, reduced their workload, enhanced the precision ratio of recognizing such calls, intercepted intelligently nuisance calls and blocked fully strange call numbers in the prentise of limited blacklist capacity.

Sampling information of telephone exchange data is summarized in Table 2. Ao facilitate the experiment and test, we sorted out three morning hours' exchange data on -one working day from exchange information of some prefecture-level city which takes up approx. $3 \%$ of total volume on the day.

Table 2. Abstract of Sampled Data

\begin{tabular}{|c|c|}
\hline Summary data & 3945190 \\
\hline Call number & 893202 \\
\hline Telephone number & 16233 \\
\hline Relates to the city regions & 7441 \\
\hline Phone number back to dial the nunber & \\
\hline
\end{tabular}

\subsection{Analysis of Telecom Business Application}

Based on week to estimate the life circle of strange calls. Together with the help of harassment frequency and instance time, we can calculate the priority of being held. The first 100 blacklist numbers from other provinces in the priority ranking list, blacklist numbers in all networks and the bife cycle are directed to webmaster interception system for barring. A few characteristios of nuisance call behavior information are extracted and the related data warehouse classification recognition model is created. Then, data are pre-treated with the proposed method. Through calculation to judge accurately the suspicious strange call numbers, the rate can reach over $80 \%$. The traditional classifying method uses mainly static attributes, negligence of return of such calls. Owing to behavioral pattern difference, features of those harassments are not carefully concerned. That's why we proposed the new method to analyze data relating to such call behavior, allocating phone numbers with the similar behavior features to one class:

(1) One side calling the other and holding time over $1.5 \mathrm{mins}$;

(2) One side calling the other but ever answered;

(3) One side calling the other but hanging up instantly;

In return, we conclude these behavior features of the other side:

(4)Unanswered call but dial-back and talk time over 1.5mins;

(5)Unanswered nor call back;

(6)Unanswered but dial-back and hang-up after 30 seconds;

(7)Answered but immediate hang-up;

(8)Answered and conversation time over $1.5 \mathrm{mins}$ 
According to the above classification, we can positively figure out suspicious strange call blacklist. The whole procedure is described as follows:

Step 1: Give M variables in $N \times M$ data matrix and partition call behavior of all numbers into $\mathrm{K}$ classes. Set $\mathfrak{R}_{i}=\left(\delta_{i, 1}, \delta_{i, 2}, \ldots, \delta_{i, m}\right)$ the weight of variable in the $i$ - $t h$ class; set $\mathfrak{R}=\left(\mathfrak{R}_{1}, \mathfrak{R}_{2}, \ldots, \mathfrak{R}_{K}\right)$ the weight collection of all variables in all $\mathrm{K}$ classes. In the instance, $\mathrm{M}=24 ; \mathrm{N}=893202$. From call behavior of those numbers in different time, we can decide the total number of classes is 8 . Set clustering parameter $\mathrm{K}=8$.

Step 2: Dynamic behavior data are normalized as to establish data matrix, such as call behavior, in-coming time, prefectural city area and talk time of all phone numbers.

Step 3: Use the proposed algorithm based on multi-rule constraint to cluster the classifying method of phone numbers, which can be realized through classification model formula 1.

Step 4: According to formula 2, the value of $\eta$ can be defined. Set feature werght $m$ this process, for the call behavior in the above viii, the weight of (3) and (7) is bigger

Step 5: Based on call behavior similarity, all phone numbers are clustered into one class as per their similarity percentage, to form different clusters.

Clustering results are seen in Figure 1, where horizontal axis is classification $\mathrm{K}$ of phone numbers; vertical axis is quantity of phone numbers included in each class. The bar chart displays the status of all original static classification information after dynamic clustering results are analyzed.

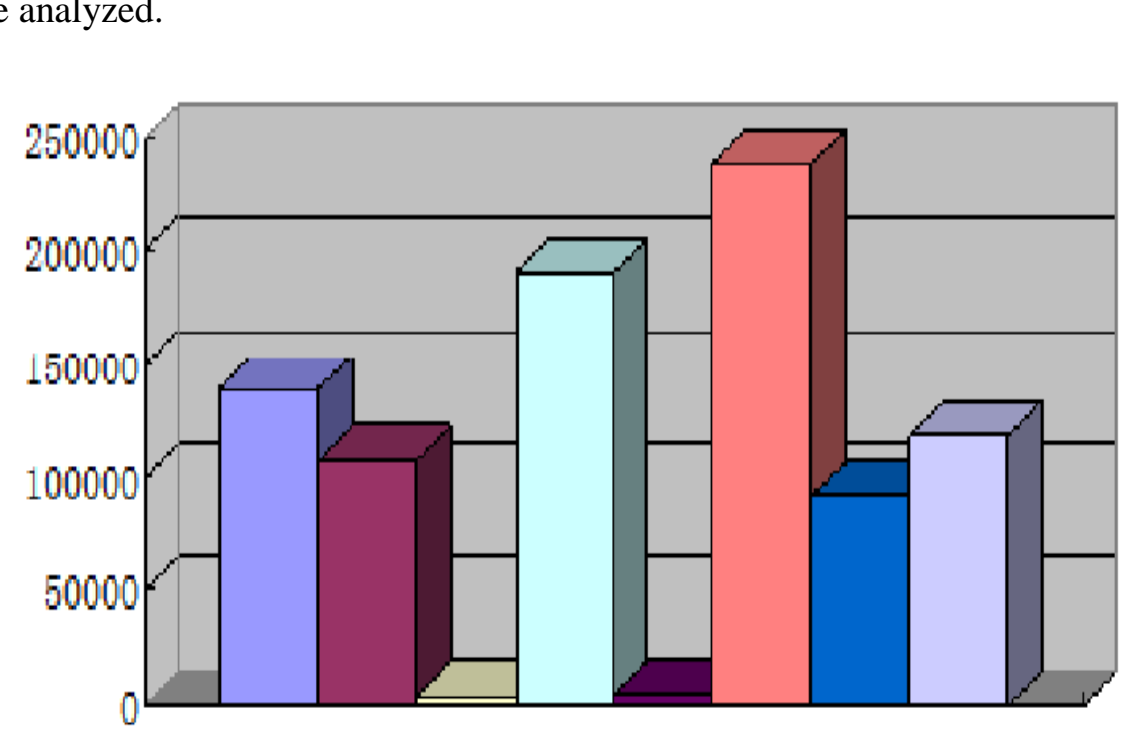

Figure 1. Results of classification algorithm

For phone numbers formed from (3) and (7), an effective discernment model can be set up and the b acklist is produced to be used in the nuisance phone number monitoring system. Therefore, it's necessity to change business operation decision in accordance to results of classification (Table 3).

Table 3. Countermeasures Classification

\begin{tabular}{|c|c|}
\hline Cluster number & Countermeasures \\
\hline $1,4,8$ & maintain the original management strategy \\
\hline 2,5 & Adopt the same management strategy \\
\hline $3,6,7$ & Further dividing the types, targeted management \\
\hline
\end{tabular}


Through strategy adjustment, the monitoring blacklist of harassment phone numbers can be formed and refined to improve the accuracy of judgment. As seen in Figure 2, the proposed method is employed to do knowledge discovery of uncertain strange phone numbers, with the accuracy ratio of that determination more than $90 \%$.

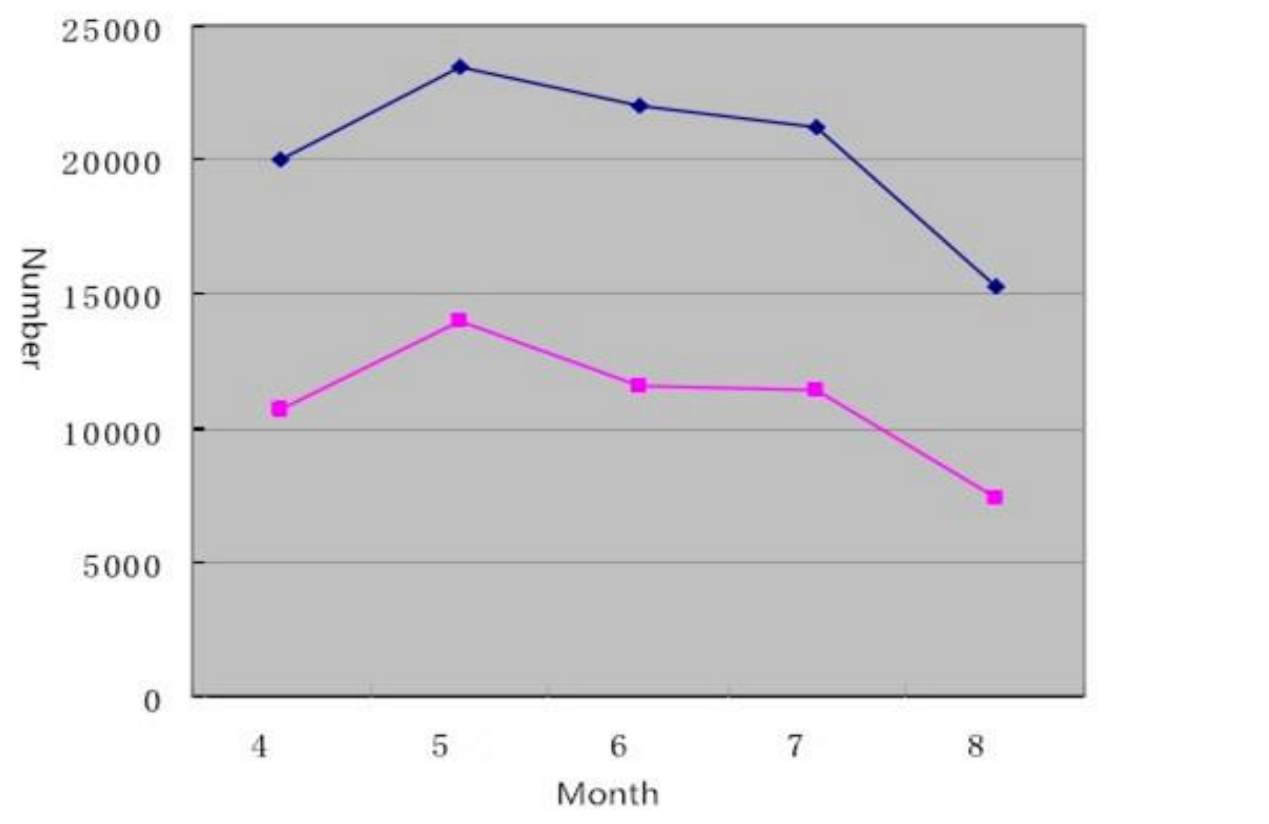

Figure 2. Statistical Number of Monitoring Harassing Phone Calls

In Figure 2, the quantity of harassment phone numbers becomes fewer and fewer since May, at $11.63 \%$ per month on average, time for discerning reduced by $963.5 \mathrm{mins}$ per capita. The subspace clustering technique based on multi-rule constraint, the detection ratio of strange calls is remarkably raised. The rate of complaints by call-back phones is hugely decreased.

\subsection{Data Magnitude Test and Analysis}

As observed from Table 4 , to prove the accuracy of the proposed method here, we compared it with traditional K-means algorithm on clustering results. The test has two phases.

Stage 1: We chose methods which only have initial clustering center optimization strategy but variable weighting function to make comparison, shown in Table 4 (a);

Stage 2: We chose complete subspace clustering algorithm based on multi-rule constraint to make comparison, listed in Table4 (b).

What's examined by those algorithms is largely degree of difference. When two methods are 101 calculation, and one object is assigned to different clusters, the object is called differential sample. The ratio of differential samples in the total samples is called the degree of difference. 
Table 4. (a) Results Comparison between Proposed Algorithm and K-means for $1^{\text {st }}$ Phase

\begin{tabular}{|l|l|l|l|l|l|l|l|l|l|l|l|}
\hline & S1 & S2 & S3 & S4 & S5 & S6 & S7 & S8 & S9 & S10 & Total \\
\hline $\begin{array}{l}\text { Total number } \\
\text { of samples }\end{array}$ & 910 & 870 & 620 & 1011 & 768 & 834 & 697 & 881 & 813 & 948 & 8352 \\
\hline $\begin{array}{l}\text { Number of } \\
\text { Differences } \\
\text { samples }\end{array}$ & 30 & 42 & 36 & 44 & 28 & 42 & 26 & 34 & 40 & 62 & 384 \\
\hline $\begin{array}{l}\text { Difference } \\
\text { degree (\%) }\end{array}$ & 3.3 & 4.8 & 5.8 & 4.3 & 3.6 & 5.0 & 3.7 & 3.8 & 4.9 & 6.5 & 4.6 \\
\hline
\end{tabular}

Table 4. (b) Results Comparison between Proposed Algorithm and K-means for $2^{\text {nd }}$ Phase

\begin{tabular}{|l|l|l|l|l|l|l|l|l|l|l|l|}
\hline & S1 & S2 & S3 & S4 & S5 & S6 & S7 & S8 & S9 & S10 & Total \\
\hline $\begin{array}{l}\text { Total number } \\
\text { of samples }\end{array}$ & 910 & 870 & 620 & 1011 & 768 & 834 & 697 & 881 & 813 & 948 & 8352 \\
\hline $\begin{array}{l}\text { Number of } \\
\text { Differences } \\
\text { samples }\end{array}$ & 7 & 12 & 7 & 21 & 15 & 7 & & 11 & 18 & 23 & 145 \\
\hline $\begin{array}{l}\text { Difference } \\
\text { degree (\%) }\end{array}$ & 0.8 & 1.4 & 2.7 & 2.0 & 1.9 & 0.8 & 1.5 & 1.5 & 2.2 & 2.4 & 1.7 \\
\hline
\end{tabular}

\subsection{Performance Validation and Analysis}

Here we chose Data and Iris which arre eorresponding to simulation and benchmark dataset to confirm the integrated performance of the proposed method. Table 5 presents the size, dimension and cluster size of dataset Iris and Data.

\section{Table 5. Descriptions of Data Sets}

\begin{tabular}{|l|l|l|l|l|}
\hline \multicolumn{1}{|c|}{ Name } & Size & The number of levels & Dimension & \multicolumn{1}{c|}{ Class size } \\
\hline Data & 1000 & 4 & 4 & $250,250,250,250$ \\
\hline Iris & 1500 & 3 & 3 & $500,500,500$ \\
\hline
\end{tabular}

Figure 3 shows the distribution of data set. Dimensional characteristics of Iris dataset makes it impossible to show a chart form. The collection contains more than 150 models, each model contains 4 attyibutes. 


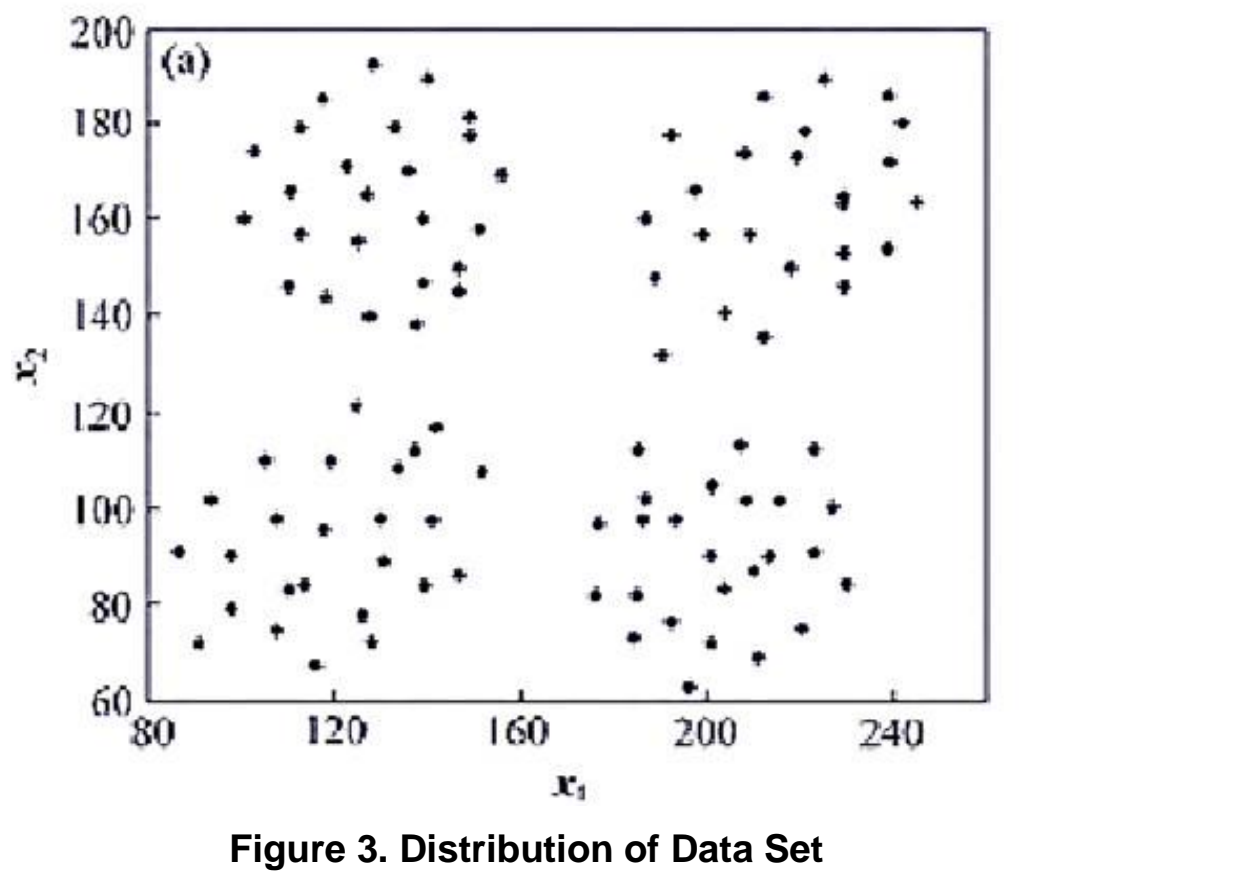

For the traditional K-means method, comparisons were made between the optimized method for the initial clustering center and subspace clustering method based on multi-rule constraint. Each was computed for three times. After comparisons, we found:

The proposed method increased apparently the total number of clustering classes;

The optimized method for the initial clustering center presents more clear structure of clustering classes than the traditional K-means method.

Table 6. Experimental Analysis for Three Kinds of Algorithms

\begin{tabular}{|l|l|l|l|l|}
\hline \multicolumn{1}{|c|}{ Algorithm } & Data set & Run time (s) & Accurate rate & $\begin{array}{l}\text { Find the number } \\
\text { of levels }\end{array}$ \\
\hline K means algorithm & Data Iris & $24.30-26.43$ & $90.28 \%$ & $4,3,3,2,3,2$ \\
\hline $\begin{array}{l}\text { The initial cluster center. } \\
\text { K means method }\end{array}$ & Data Iris & $12.22-13.98$ & $94.81 \%$ & $4,4,4,3,3,3$ \\
\hline $\begin{array}{l}\text { Multi rule constraint subspace } \\
\text { Clustering algorithn }\end{array}$ & Data Iris & $9.02-11.64$ & $97.73 \%$ & $4,4,4,3,3,3$ \\
\hline
\end{tabular}

Table 6 is the test environment three kinds of clustering algorithm in the data set standards under the experimental analysis, Table 5 gives the average running time and accuracy.

\subsection{Enulation Test}

For the emulation, we selected traditional K-means algorithm, GA-based K-means method and subspace clustering method proposed here. The experiment used VC development in MATLAB environment. In the test, for the genetic algorithm, we set crossover probability 0.76 , mutation possibility 0.08 , group size 1000 and 100 iterations. When the number to obtain the best result is consecutively up to 10 times, it's over. The test dataset is still Iris, of 150 samples. Suppose every method of the three run 10 times to get the maximum, minimum 
and mean values in the intra-cluster distance. On that basis, we calculated the maximum, minimum and mean values in the inter-cluster distance. Results are all seen in Table 7.

Table 7. Experimental Results for Iris Data Sets

\begin{tabular}{|l|l|l|l|l|l|l|c|}
\hline Algorithm & $\begin{array}{l}\text { The } \\
\text { maximum } \\
\text { distance } \\
\text { cluster }\end{array}$ & $\begin{array}{l}\text { The } \\
\text { minimum } \\
\text { distance } \\
\text { cluster } \\
\text { average } \\
\text { distance } \\
\text { of } \\
\text { clusters }\end{array}$ & $\begin{array}{l}\text { The } \\
\text { maximum } \\
\text { distance } \\
\text { between } \\
\text { clusters }\end{array}$ & $\begin{array}{l}\text { The } \\
\text { minimum } \\
\text { distance } \\
\text { between } \\
\text { clusters }\end{array}$ & $\begin{array}{l}\text { The } \\
\text { average } \\
\text { distance } \\
\text { between } \\
\text { clusters }\end{array}$ & $\begin{array}{l}\text { Optimal } \\
\text { iteration }\end{array}$ \\
\hline $\begin{array}{l}\text { K means } \\
\text { algorithm }\end{array}$ & 13.392 & 9.492 & 11.832 & 9.201 & 5.081 & 5.920 & 46 \\
\hline $\begin{array}{l}\text { K means } \\
\text { algorithm } \\
\text { based on } \\
\text { genetic } \\
\text { algorithm }\end{array}$ & 13.209 & 9.313 & 11.642 & 9.892 & 5.293 & 6.082 & 37 \\
\hline $\begin{array}{l}\text { This paper } \\
\text { algorithm }\end{array}$ & 13.002 & 7.498 & 11.105 & 10.174 & & & \\
\hline
\end{tabular}

For clustering methods, the goal is to obtain both the minimum intra-cluster distance and the maximum inter-cluster distance. From Table 7 , traditional K-means method depends more on the selection of initial center for the accuracy of clustering. Once it's selected wrong, it will fall into local optimum, leading to earlier convergence. For GA-based K-means method, too simplified data and worse diversity caused pre-mature, disable to cope with the problem of data sparseness. The proposed method keeps the search ability of genetic algorithm in global area and has the power to solve sparse datato acquire data of higher quality.

\section{Conclusion}

This paper analyses the defects and core problem in traditional $\mathrm{K}$ means methods. That is difficult to select the mitial cluster centers and data processing classification sparse case. For these two problems, we proposed corresponding solutions: to achieve the global optimal search using the genetic algerithm, to avoid falling into local optimum clustering results. Improved subspace clustering strategy using the variable weighted, it can handle massive high dimensional sparse data. On this basis, the proposed method makes a practical analysis combined with telecom specific business, and compares it with the traditional clustering algorithm, the performance of the paper algorithm are verified the accuracy and rationality.

\section{References}

[1] Y. Xiong, "Analysis of the telecommunications industry management system construction discussion", The ecounting \&amp;OSS world, no. 11, (2004), pp. 43-44.

[2] B Rongbing, "Research and application of grid density based on data mining", Master Thesis of Nanjing Institute of meteorology, (2003), pp. 12-19.

[3] O. Grupe, "DATA BASE MINING", Discovering New Knowledge and Competitive Advantage, (2005), pp. 22-24.

[4] C. Ping, W. Bo and X. Liutong, "Parallel algorithm and scheduling algorithm of grid Telecom social network intermediary degree", Journal of Beijing University of Posts and Telecommunications, no. 12, (2006), pp. 3437.

[5] R. Agrawal, T. Imielinski and A. Swami, "Mining association rules between sets of items in large database", Proceeding of the 2003 ACM SIGMOD International Conference on Management of Data. New York: ACM Press, (2003), pp. 207-216. 
[6] Y. Xiang, Y. GUI, X. Xiandong and W. Jianwei, "A flow of subspace clustering method based on the data of regional division", Journal of computer research and development, vol. 01, (2014), pp. 88-95.

[7] X. Yingmei, "Research on clustering algorithm for dynamic data flow within the sliding window", Journal of Shaanxi University of Technology (NATURAL SCIENCE EDITION), vol. 01, (2014), pp. 42-46.

[8] X. Y. Xi, H. Huan, J. Jin and Y. Huaiwang, "A kind of uncertain data stream subspace clustering algorithm”, Information technology, vol. 02, (2014), pp. 27-30.

[9] L. You, L. Wei, L. Junzhou, J. Jian and X. Anger, "A selective cooperative learning network user behavior anomaly detection method", based on Chinese Journal of computers, vol. 01, (2014), pp. 28-40.

[10] W. Lijuan, H. Zhifeng, C. Ruichu and W. Wen, "Algorithm locally adaptive subspace clustering based on multi disturbances", Computer science, vol. 02, (2014), pp. 240-244.

[11] L. Weisheng, G. Gongde and C. Lifei, "SMwKnn: mutual k nearest neighbor algorithm based on weighted subspace distance", Computer science, vol. 02, (2014), pp. 166-169.

[12] L. Tao, W. Weiwei and Z. D. Jia, "Weighted sparse subspace clustering image segmentation method", Systems engineering and electronics, vol. 03, (2014), pp. 580-585.

[13] W. Ailian, W. Weili and C. Junjie, "Comparison and improved image segmentation method based on Kmeans clustering algorithm", Journal of Taiyuan University of Technology, vol. 03, (2014), pp. 372375.

[14] W. Lijuan, H. Zhifeng, C. Ruichu and W. Wen, "A real value link analysis fusion algorithm based on ESSC", The research and application of computer, vol. 05, (2014), pp. 1366-1369.

[15] S. Aihui, H. Shucheng and L. Sweet, "A clustering algorithm of netwotk community structure and module function", Based on the computer application and software, vol. 04, (2014)

Author

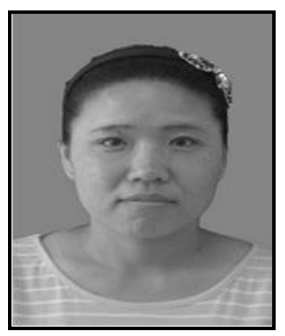

Li Huiping, she received her master's degree of engineering in Inner Mongolia Normal University. Now she is a lecturer in Bao Tou medical college. She is in the research of computer application.

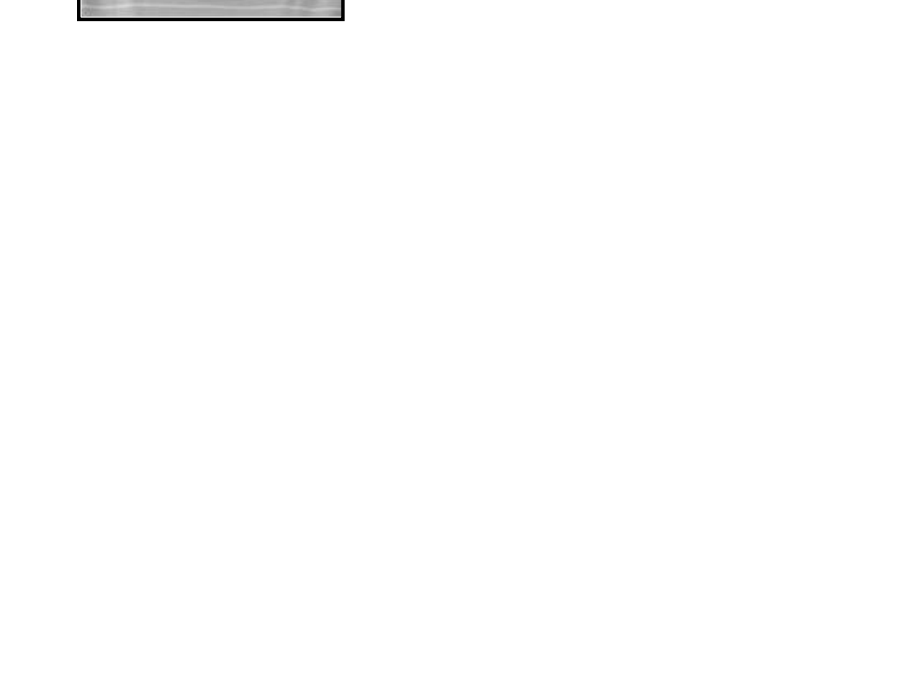

